Relations industrielles

Industrial Relations

\title{
CAMPBELL, Ralph N., "Human Relations in Industry", Reprint Series Number 15, New York State School of Industrial and Labor Relations at Cornell University, 5 pp., 1953.
}

\section{Fabienne Tousignant}

Volume 9, numéro 1, décembre 1953

URI : https://id.erudit.org/iderudit/1022921ar

DOI : https://doi.org/10.7202/1022921ar

Aller au sommaire du numéro

Éditeur(s)

Département des relations industrielles de l’Université Laval

ISSN

0034-379X (imprimé)

1703-8138 (numérique)

Découvrir la revue

Citer ce compte rendu

Tousignant, F. (1953). Compte rendu de [CAMPBELL, Ralph N., "Human Relations in Industry", Reprint Series Number 15, New York State School of Industrial and Labor Relations at Cornell University, 5 pp., 1953.] Relations industrielles / Industrial Relations, 9(1), 86-86. https://doi.org/10.7202/1022921ar

Tous droits réservés (C Département des relations industrielles de l’Université Laval, 1953
Ce document est protégé par la loi sur le droit d'auteur. L'utilisation des services d'Érudit (y compris la reproduction) est assujettie à sa politique d'utilisation que vous pouvez consulter en ligne.

https://apropos.erudit.org/fr/usagers/politique-dutilisation/ 


\section{LIVRES et RE VUES}

\section{Commission Sacerdotale d'Etudes Sociales}

Bibliographie sur l'organisation professionnelle, collection Etudes et recherches sociales, no. 4 - octobre 1953. 1 volume, 44 pages. Prix: $\$ 1.00$

Contient une liste assez complète des ouvrages publiés en langue française soit au Canada ou en France sur ce sujet. On a suivi le plan suivant: Doctrine de l'Eglise; notions générales; aspects particuliers; organisation, réalisation, bibliographies.

Secrétariat National d'Action Sociale, 1895, rue Girouard, St-Hyacinthe, P.Q.

CAMPBELL, Ralph N., "Human Relations in Industry", Reprint Series Number 15, New York State School of Industrial and Labor Relations at Cornell University, 5 pp., 1953.

L'auteur dans ce court article veut considérer l'individu en tant qu'être humain; d'après lui, la majorité des écrits portant sur le sujet des relations humaines dans l'industrie se sont arrêtés à l'individu en relation avec son travail et avec son entourage immédiat ou lointain. On peut alors être porté à penser et à agir comme si l'employé, le surveilant, le propriétaire et le chef ouvrier sont différentes sortes d'êtres humains ou que tous les individus appartenant à un groupe sont tous semblables... mais on sait que rien n'est plus faux!

Le problème des relations humaines dans l'industrie est d'obtenir de la collaborafion à tous les niveaux au moyen de gens qui s'entendent bien. Ce n'est ni simple, ni facile. En effet, l'argent seul ne peut acheter la collaboration ou l'initiative de qui que ce soit; la raison en est que les êtres humains ont certains désirs qui ne peuvent être satisfaits par l'argent seulement. Evidemment, il $y$ a certaines exigences économiques fondamentales à tous: faim, soif, chaieur, froid et fatigue. Une fois que ces besoins sont contentés, d'autres déjà se font sentir.

Les recherches conduites au Hawthorne Plant of the Western Electric Co. sur un groupe de quatorze ouvriers travaillant à la pièce et rémunérés selon la production du groupe lui-même ont permis de découvrir que certains sentiments dominaient chez-eux, entre autres:

1. Ne pas trop produire pour ne pas faire sauter le taux de la production.

2. Ne pas si peu produire pour faire baisser ce taux.

3. Ne dire à un surveilant rien qui puisse avoir des répercussions sur un des associés.

4. Ne pas être trop officieux, ainsi celui qui est inspecteur ne doit pas agir en inspecteur.

En effet, chaque personne a le besoin de se sentir, en tant qu'être humain, aussi importante que tout individu. Cela ne signifie pas que les individus s'objectent à l'autorité, au contraire, ils en voient la nécessité, mais ils demandent qu'elle soit exercée par des gens compétent's et qui ne veulent pas uniquement afficher leur importance.

L'employé demande à être renseigné de plus en plus sur la marche de l'entreprise et, en autant que possible, aime à discuter des changements qui peuvent l'affecter en quelque façon. D'ailleurs, cette méthode d'information peut' devenir la source de suggestions, d'idées de la part des travailleurs eux-mêmes. De plus, chacun aime à pouvoir travailler sur une tâche qui requerra son habileté et dans un entourage où il peut se sentir accepté par les autres.

Celui qui doit conduire des hommes doit se rappeler qu'il lui faut une grande compréhension des besoins humains, une tolérance de la faiblesse humaine et une détermination d'en venir à un compromis en respectant le bien général de tous tout en tenant compte le plus possible des intérêts et droits de chacun.

Cet article a une portée intéressante en tant qu'il permet au lecteur de découvrir certains facteurs de satisfaction du travailleur à son travail.

F. T. 